Rend. Sem. Mat. Univ. Padova 1xx (201x) Rendiconti del Seminario Matematico della Università di Padova

(C) European Mathematical Society

\title{
A harmonic mean inequality for the digamma function and related results
}

\author{
Horst Alzer - Graham Jameson
}

ABSTRACT - We present some inequalities and a concavity property of the digamma function $\psi=\Gamma^{\prime} / \Gamma$, where $\Gamma$ denotes Euler's gamma function. In particular, we offer a new characterization of Euler's constant $\gamma=0.57721 \ldots$. We prove that $-\gamma$ is the minimum of the harmonic mean of $\psi(x)$ and $\psi(1 / x)$ for $x>0$.

Mathematics Subject Classification (2010). 33B15, 39B62, 41A44.

KEYWORDS. Digamma function, inequalities, concavity, harmonic mean, Euler's constant.

\section{Introduction and main result}

In 1974, Gautschi [12] presented a very interesting mean value inequality for the classical gamma function of Euler. He proved that for all positive real numbers $x$ the harmonic mean of $\Gamma(x)$ and $\Gamma(1 / x)$ is greater than or equal to 1 , that is,

$$
1 \leq \frac{2 \Gamma(x) \Gamma(1 / x)}{\Gamma(x)+\Gamma(1 / x)} \quad(x>0) .
$$

The sign of equality holds for $x=1$.

As an immediate consequence of (1.1) we obtain the inequalities

$$
2 \leq \Gamma(x)+\Gamma(1 / x) \quad(x>0)
$$

and

$$
1 \leq \Gamma(x) \Gamma(1 / x) \quad(x>0)
$$

Horst Alzer, Morsbacher Str. 10, 51545 Waldbröl, Germany

E-mail: h.alzer@gmx.de

Graham Jameson, Department of Mathematics and Statistics, Lancaster University, Lancaster LA1 4YF, UK.

E-mail: g.jameson@lancaster.ac.uk 
In several research papers numerous refinements, extensions and relatives of Gautschi's inequality were published; see [3], [4], [5], [6], [8], [9], [13], [14], [15]. In this article we offer a counterpart of (1.1) for the digamma (or psi) function

$$
\psi(x)=\frac{\Gamma^{\prime}(x)}{\Gamma(x)}=\int_{0}^{\infty}\left(\frac{e^{-t}}{t}-\frac{e^{-x t}}{1-e^{-t}}\right) d t=-\gamma-\frac{1}{x}+\sum_{k=1}^{\infty} \frac{x}{k(x+k)} \quad(x>0) .
$$

Here, $\gamma=-\psi(1)=0.57721 \ldots$ denotes Euler's constant.

The $\psi$-function is strictly increasing on $(0, \infty)$ with $\psi\left(x_{0}\right)=0$, where $x_{0}=$ $1.46163 \ldots$ In particular, $\psi$ is negative on $\left(0, x_{0}\right)$ and positive on $\left(x_{0}, \infty\right)$. A collection of the most important properties of this function can be found, for instance, in [1, chapter 6$]$ and [18, chapter 5].

The digamma function plays an important role in several branches of mathematics and related areas. In fact, it has interesting applications in the theory of special functions, in the theory of infinite series, in statistics, in physics and it is also subject of number theoretic investigations. See [10], [11], [16], [17], [19].

It is our main goal to prove the following striking companion of (1.1).

THEOREM. For all positive real numbers $x$ we have

$$
-\gamma \leq \frac{2 \psi(x) \psi(1 / x)}{\psi(x)+\psi(1 / x)}
$$

The sign of equality holds if and only if $x=1$.

This result leads to a new characterization of Euler's constant: $-\gamma$ is the minimum of the harmonic mean of $\psi(x)$ and $\psi(1 / x)$ for $x>0$. Since the expression on the right-hand side of (1.4) tends to $\infty$ if $x \rightarrow 0^{+}$, we conclude that there is no constant upper bound which is valid for all $x>0$.

In the next section we present a concavity property of the digamma function and some inequalities. With the help of these results we are able to offer a short proof of the Theorem. This proof is given in Section 3.

\section{Concavity and inequalities}

It is well-known that the $\psi$-function is strictly concave on $(0, \infty)$. Since $x \mapsto$ $x^{2} \psi^{\prime}(x)$ is strictly increasing on $(0, \infty)$, see [7], we conclude from

$$
\frac{d}{d x} \psi(1 / x)=-\frac{1}{x^{2}} \psi^{\prime}(1 / x) \quad(x>0)
$$

that $x \mapsto \psi(1 / x)$ is strictly convex on $(0, \infty)$. Now we study $x \mapsto \psi(x)+\psi(1 / x)$.

Proposition 1. The function

$$
P(x)=\psi(x)+\psi(1 / x)
$$

is strictly concave on $(0, \infty)$. 
Proof. By differentiation we find

$$
x^{4} P^{\prime \prime}(x)=2 x \psi^{\prime}(1 / x)+\psi^{\prime \prime}(1 / x)+x^{4} \psi^{\prime \prime}(x) .
$$

Applying the recurrence relations

$$
\psi^{\prime}(x+1)=\psi^{\prime}(x)-\frac{1}{x^{2}}, \quad \psi^{\prime \prime}(x+1)=\psi^{\prime \prime}(x)+\frac{2}{x^{3}}
$$

and the estimates

$$
\psi^{\prime}(x)<\frac{1}{x}+\frac{1}{2 x^{2}}+\frac{1}{6 x^{3}}, \quad \psi^{\prime \prime}(x)<-\frac{1}{x^{2}}-\frac{1}{x^{3}} \quad(x>0)
$$

which are given in [2], we obtain for positive $x$ :

$$
\begin{aligned}
x^{4} P^{\prime \prime}(x)= & 2 x\left(x^{2}+\psi^{\prime}(1+1 / x)\right)-2 x^{3}+\psi^{\prime \prime}(1+1 / x)+x^{4} \psi^{\prime \prime}(x) \\
< & 2 x\left(x^{2}+\frac{1}{1+1 / x}+\frac{1}{2(1+1 / x)^{2}}+\frac{1}{6(1+1 / x)^{3}}\right) \\
& \quad-2 x^{3}-\frac{1}{(1+1 / x)^{2}}-\frac{1}{(1+1 / x)^{3}}+x^{4}\left(-\frac{1}{x^{2}}-\frac{1}{x^{3}}\right) \\
= & -\frac{x}{3(1+x)^{3}}\left(3 x^{4}+2 x^{3}+9 x^{2}+9 x+3\right) \\
< & 0 .
\end{aligned}
$$

This reveals that $P$ is strictly concave on $(0, \infty)$.

An application of Proposition 1 leads to the following analogue of (1.2).

Proposition 2. For all positive real numbers $x \neq 1$ we have

$$
\psi(x)+\psi(1 / x)<-2 \gamma .
$$

The upper bound is sharp.

Proof. Since $P^{\prime \prime}$ is negative on $(0, \infty)$, we obtain $P^{\prime}(x)>P^{\prime}(1)=0$ for $x \in(0,1)$ and $P^{\prime}(x)<P^{\prime}(1)=0$ for $x>1$. It follows that $P$ is strictly increasing on $(0,1]$ and strictly decreasing on $[1, \infty)$. Thus $P(x)<P(1)=-2 \gamma$ for $x>0, x \neq 1$.

REMARK 1. Inequality (2.1) can also be proved by using the fact that the function $u(x)=x \psi^{\prime}(x)$ is strictly decreasing on $(0, \infty)$. Indeed, since $x P^{\prime}(x)=$ $u(x)-u(1 / x)$, we obtain that $P^{\prime}$ is positive on $(0,1)$ and negative on $(1, \infty)$. A proof for the monotonicity behaviour of $x \mapsto x^{c}\left|\psi^{(k)}(x)\right|(c \in \mathbb{R} ; k \in \mathbb{N})$ is given in $[7]$.

Next, we present two inequalities which are closely related to (2.1). 
Proposition 3. For all real numbers $y \in(0,1)$ we have

$$
\psi(1+y) \psi(1-y)<\gamma^{2}
$$

The upper bound is sharp.

Proof. If $y \in\left[x_{0}-1,1\right)$, then we have $\psi(1-y)<0 \leq \psi(1+y)$. This reveals that $(2.2)$ holds. Next, we assume that $y \in\left(0, x_{0}-1\right)$. Using the power series expansion

$$
\psi(1+z)=-\gamma+\sum_{k=2}^{\infty}(-1)^{k} \zeta(k) z^{k-1} \quad(|z|<1)
$$

we obtain the estimates

$$
0<-\psi(1+y) \leq \gamma-\zeta(2) y+\zeta(3) y^{2}
$$

and

$$
\begin{aligned}
0<-\psi(1-y) & \leq \gamma+\zeta(2) y+\zeta(3) \sum_{k=2}^{\infty} y^{k} \\
& =\gamma+\zeta(2) y+\zeta(3) \frac{y^{2}}{1-y} \\
& \leq \gamma+\zeta(2) y+2 \zeta(3) y^{2}
\end{aligned}
$$

Thus

$$
\psi(1+y) \psi(1-y) \leq \gamma^{2}-c_{2} y^{2}-c_{3} y^{3}+c_{4} y^{4}
$$

with

$c_{2}=\zeta(2)^{2}-3 \gamma \zeta(3)=0.624 \ldots, \quad c_{3}=\zeta(2) \zeta(3)=1.977 \ldots, \quad c_{4}=2 \zeta(3)^{2}=2.889 \ldots$

We have $c_{3}>c_{4} / 2$. Hence $c_{3} y^{3}>c_{4} y^{4}$. This leads to $(2.2)$.

Since

$$
\lim _{y \rightarrow 0} \psi(1+y) \psi(1-y)=\gamma^{2}
$$

we conclude that the upper bound given in (2.2) is sharp.

The following counterpart of (1.3) is valid.

Proposition 4. For all positive real numbers $x \neq 1$ we have

$$
\psi(x) \psi(1 / x)<\gamma^{2}
$$

The upper bound is sharp. 
Proof. It suffices to prove (2.3) for $x>1$. If $x \geq x_{0}$, then $\psi(1 / x)<0 \leq \psi(x)$. It follows that $(2.3)$ is valid.

Next, let $x \in\left(1, x_{0}\right)$. We set $x=1+y$. Then $1 / x>1-y$. Thus $\psi(1-y)<$ $\psi(1 / x)$. Applying this inequality and (2.2) yields

$$
\psi(x) \psi(1 / x)=\psi(1+y) \psi(1 / x)<\psi(1+y) \psi(1-y) \leq \gamma^{2} .
$$

Since $\psi(1)=-\gamma$, the upper bound given in (2.3) is best possible.

REMARK 2. The limit relations

$$
\begin{gathered}
\lim _{x \rightarrow 0^{+}} \psi(x)+\psi(1 / x)=-\infty, \quad \lim _{y \rightarrow 1^{-}} \psi(1+y) \psi(1-y)=-\infty, \\
\lim _{x \rightarrow 0^{+}} \psi(x) \psi(1 / x)=-\infty
\end{gathered}
$$

reveal that there are no constant lower bounds for the expressions given in (2.1), (2.2) and (2.3) respectively.

\section{Proof of Theorem}

From (2.1) we conclude that the expression on the right-hand side of (1.4) is defined for all positive $x$. Applying (2.1) and (2.3) gives for $x>0, x \neq 1$ :

$$
2 \psi(x) \psi(1 / x) \cdot \frac{1}{\psi(x)+\psi(1 / x)}>2 \gamma^{2} \cdot \frac{1}{\psi(x)+\psi(1 / x)}>2 \gamma^{2} \cdot \frac{1}{-2 \gamma}=-\gamma .
$$

The proof of the Theorem is complete.

\section{REFERENCES}

[1] M. Abramowitz, I.A. Stegun (eds.), Handbook of Mathematical Functions with Formulas, Graphs, and Mathematical Tables, Dover, New York, 1992.

[2] H. Alzer, On some inequalities for the gamma and psi functions, Math. Comp. 66 (1997), pp. 373-389.

[3] H. Alzer, A harmonic mean inequality for the gamma function, J. Comp. Appl. Math. 87 (1997), pp. 195-198.

[4] H. Alzer, Inequalities for the gamma function, Proc. Amer. Math. Soc. 128 (1999), pp. 141-147.

[5] H. Alzer, On a gamma function inequality of Gautschi, Proc. Edinburgh Math. Soc. 45 (2002), pp. 589-600.

[6] H. Alzer, On Gautschi's harmonic mean inequality for the gamma function, J. Comp. Appl. Math. 157 (2003), pp. 243-249.

[7] H. Alzer, Mean-value inequalities for the polygamma functions, Aequat. Math. 61 (2001), pp. 151-161. 
[8] H. Alzer, Inequalities involving $\Gamma(x)$ and $\Gamma(1 / x)$, J. Comp. Appl. Math. 192 (2006), pp. $460-480$.

[9] H. Alzer, Gamma function inequalities, Numer. Algor. 49 (2008), pp. 53-84.

[10] M.C. Coffey, On one-dimensional digamma and polygamma series related to the evaluation of Feynman diagrams, J. Comp. Appl. Math. 183 (2005), pp. 84-100.

[11] P.J. de Doelder, On some series containing $\psi(x)-\psi(y)$ and $(\psi(x)-\psi(y))^{2}$ for certain values of $x$ and $y$, J. Comp. Appl. Math. 37 (1991), pp. 125-141.

[12] W. Gautschi, A harmonic mean inequality for the gamma function, SIAM J. Math. Anal. 5 (1974), pp. 278-281.

[13] W. Gautschi, Some mean value inequalities for the gamma function, SIAM J. Math. Anal. 5 (1974), pp. 282-292.

[14] C. Giordano, A. Laforgia, Inequalities and monotonicity properties for the gamma function, J. Comp. Appl. Math. 133 (2001), pp. 387-396.

[15] G.J.O. Jameson, T.P. Jameson, An inequality for the gamma function conjectured by D. Kershaw, J. Math. Ineq. 6 (2012), pp. 175-181.

[16] M.R. Murty, N. Saradha, Transcendental values of the digamma function, J. Number Th. 125 (2007), pp. 298-318.

[17] O.M. Ogreid, P. Osland, Some infinite series related to Feynman diagrams, J. Comp. Appl. Math. 140 (2002), pp. 659-671.

[18] F.W.J. Olver, D.W. Lozier, R.F. Boisvert, C.W. Clark (eds.), NIST Handbook of Mathematical Functions, Cambridge Univ. Press, New York, 2010.

[19] N.M. Temme, Special Functions: An Introduction to the Classical Functions of Mathematical Physics, Wiley, New York, 1996.

Received submission date; revised revision date 